\title{
Análisis del índice de rugosidad internacional. Aplicación al aeropuerto Enrique Olaya Herrera de Medellín, Colombia
}

\section{Analysis of international roughness index. Application to Enrique Olaya Herrera Airport in Medellín, Colombia}

\section{Didier Marín Montoya* Mónica Riscanevo López* Pedro Mauricio Acosta Castellanos**}

\footnotetext{
* Especialización en geotécnia vial y pavimentos, Universidad Santo Tomás Seccional Tunja.

** Grupo de investigación ACIB, Universidad Santo Tomás Seccionall Tunja,
}

\section{Resumen}

El índice de rugosidad internacional surge como una necesidad de unificar mundialmente los criterios para medir la calidad del pavimento terminado en donde la gestión del transporte en un aspecto muy sensible dentro del sistema logístico y un error puede significar importantes consecuencias económicas, es por esto que se ha optado por concesionar varias vías a nivel nacional y más recientemente varios de los aeropuertos del país. Una de estas concesiones es la regional Centro Norte que administra la empresa Airplan y de la cual hace parte el aeropuerto Enrique Olaya Herrera de la ciudad de Medellín. El término rugosidad se refiere a las imperfecciones de la superficie de rodadura que inciden en la velocidad, costos de operación, seguridad vial y la comodidad de los usuarios al circular por los pavimentos, sin embargo, en el contexto de este artículo se utilizará el término regularidad superficial como un calificativo más adecuado.

El análisis tiene como objeto mostrar que esta metodología puede ser adoptada como criterio de aceptación o rechazo en las pistas aeroportuarias dadas en concesión en nuestro país y de esta manera se pueda garantizar a los usuarios un determinado nivel de seguridad, comodidad y eficiencia en las pistas.

Palabras clave: Aeropuertos, índice de regularidad internacional, pavimentos, transporte aéreo.

\begin{abstract}
The international roughness index surges as the need to worldwide unify criteria to measure terminated pavement quality, where transportation management is a very critical aspect within the logistic system, and where any error may result in great economic consequences; therefore, several roadways have been awarded nationwide, and more recently, several airports of Colombia. One of these concessions is the Center North
\end{abstract}

Recibido: 02/02/2016

Revisado: 13/03/2016

Aceptado: 12/12/2016

Correspondencia de autor: didier.marin@usantoto.edu.co monica.riscanevo@usantoto.edu.co pedro.acosta@usantoto.edu.co

(C) 2016 Universidad La Gran Colombia. Este es un artículo de acceso abierto, distribuido bajo los términos de la licencia Creative Commons Attribution License, que permite el uso ilimitado, distribución y reproducción en cualquier medio, siempre que el autor original y la fuente se acrediten.

\section{Cómo citar:}

Marín,M.D.,Riscanevo,L. M., Acosta, C.P.(2016)Análisis del índice de rugosidad internacional. Aplicación al aeropuerto Enrique Olaya Herrera de Medellín, Colombia, UGCiencia 22, 57-69.

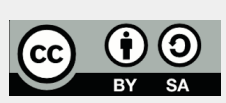


regional, managed by Airplan company, and which includes Enrique Olaya Herrera airport, in Medellin city. The term 'roughness' refers to imperfections of rolling surface, which impact velocity, operation cost, roadway safety, and user comfort when circulating on pavement; however, within the context of this article, the term 'surface regularity' will be used as a more suitable adjective. The purpose of the analysis is to show that this method may be adopted either as an acceptation or rejection criterion in airstrips awarded on concession in Colombia, and in this manner ensure users a determined level of safety, comfort, and airstrip efficiency.

Key words: Airports, national regularity index, pavements, air transportation.

\section{Introducción}

El transporte es entendido como el desplazamiento de personas y mercancías a lo largo del espacio físico mediante tres modos principales, terrestre, aéreo, y marítimo o la combinación de los mismos. (Duque Escobar, 2007).

El transporte aéreo contribuye de forma importante a la prosperidad económica mundial. La aviación es una parte vital de una economía mundial cada vez más globalizada, facilitando el crecimiento del comercio internacional, el turismo y la inversión internacional, y conectando a personas en todos los continentes. Desempeña un papel vital en facilitar el crecimiento económico, particularmente en los países en desarrollo. ( Air Transport Action Group, 2012).

En la economía colombiana, el transporte aéreo es uno de los servicios más importantes dado que da solución a la conexión de zonas que por su topografía, son difíciles de acceder con otros modos de transporte, llegando a algunas de las regiones más apartadas, de igual forma conectando a nuestro país con el resto del mundo permitiendo que haya una interacción con la economía global. (Olivera, Cabrera,
Bermúdez, \& Hernández, 2011). En Colombia los aeropuertos concesionados transportan el mayor volumen de pasajeros que se embarcan en las distintas ciudades del país, motivo por el cual deben encontrarse en condiciones óptimas de infraestructura para ofrecer un buen servicio.

La Aeronáutica Civil es la entidad que regula los aeropuertos de mayor tráfico en el país; con el ánimo de obtener una administración público-privada de los aeropuertos, se estableció en Colombia, a través de la AeroCivil, el Instituto Nacional de Concesiones (Inco) y la Agencia Nacional de Infraestructura (ANI), el sistema de otorgamiento de concesiones a empresas o consorcios para que estos se encarguen por su cuenta y riesgo de la administración, modernización, expansión, operación, explotación comercial y mantenimiento del área concesionada. (Durán Preciado, 2013).

Sin embargo, se requieren grandes inversiones económicas para garantizar la adecuada operación de estos terminales aéreos, en los últimos años el aumento de la demanda por viajes de pasajeros y carga ha implicado que se realicen esfuerzos económicos importantes para mejorar la infraestructura de los aeropuertos 
y terminales de carga aérea, llevando a que se hayan presentado inversiones totales en el sector aéreo para 2012 de 308.8 USD millones, cifra 56\% mayor que los 197.97 USD que se invirtieron en 2011 (Durán Preciado, 2013).

Dentro de la infraestructura que compone un aeropuerto, la pista de aterrizaje y las calles de conexión son las mas importantes, ya que de la regularidad de ellas depende en gran parte la seguridad y el confort de los usuarios (Sánchez \& de Solminihac, 1989). Seguridad y comodidad son parámetros empleados para evaluar el servicio, por lo que dentro de las labores de construcción, mantenimiento, reparación o reconstrucción de las aeropistas debiera considerarse un indice que permita controlar estas condiciones.

Tal es el caso del Índice de Regularidad Internacional que permite especificar rangos o niveles de tolerancia para la aceptación de tramos nuevos de aeropistas y carreteras, sirviendo como un parámetro de control de calidad superficial (Shain, 1982) y así monitorear el comportamiento de la vía a través del tiempo permitiendo fijar umbrales de alerta para proceder a un estudio de los daños o para realizar las labores de mantenimiento de acuerdo con la importancia del camino. Sin embargo, no solamente es de tener en cuenta las condiciones de seguridad y confort, sino que además se pueda cuantificar el estado en el que se encuentran los pavimentos para tomar las acciones correctivas con un tiempo pertinente.

Los sistemas de gestión de pavimentos son herramientas útiles en la definición de estrategias de mantenimiento o rehabilitación, teniendo en cuenta los costos y las condiciones de serviciabilidad de los mismos. La implementación de estos sistemas de gestión para la evaluación de los daños de las estructuras de pavimentos en los aeropuertos se centran en consideraciones estructurales, comolalimitación de tensiones, deformaciones y deflexiones en una o más capas (Monischmit, 1977). Desde hace ya algunos años se ha reconocido que la evaluación del pavimento también debe hacerse desde el punto de vista funcional (Witczak, 1978). Indicando que la estructura debe brindar condiciones de seguridad y comodidad a los usuarios, factores importantes en el campo de la aviación, especialmente relacionado con las maniobras aire-tierra-aire en donde un buen acabado superficial del pavimento conduce a una operación suave y segura de las aeronaves.

En el aeropuerto Enrique Olaya Herrera de Medellin, que esta a cargo del concesionario Ariplan, actualmente se estan realizando inversiones en infraestructura, especialmente en la re-pavimentación de la pista principal, con el objetivo de elevar la calificación del índice de estado de la misma, no obstante, dentro de los criterios de aceptación de los trabajos no se tiene en cuenta el índice de regularidad internacional, ya que la normativa adoptada para los aeropuertos en Colombia es la de la Federal Aviation Administration o FAA, entidad gubernamental responsable de la regulación de todos los aspectos de la aviación civil en los Estados Unidos y en estas no está considerado como un criterio de aceptación o rechazo el IRI.

De acuerdo con lo anterior surge la necesidad de que para las pistas de aeropuertos en Colombia, se introduzca la medición del índice de regularidad superficial como criterio de aceptación de las diferentes obras en las aeropistas, para que de esta manera se detecten a tiempo y de forma acertada los daños en la estructura del pavimento, que más adelante resultarían más costosos y traumáticos al momento de reparar y a su vez se garanticen la seguridad y confort de los usuarios. 


\section{Materiales y métodos}

\section{A. Aspectos generales}

En las últimas décadas el transporte ha sido un sector con un amplio crecimiento en el país, llegando al punto en que se han aumentado las operaciones aéreas, debido a las grandes demandas el tamaño y peso de los aviones también ha cambiado, lo cual ha traído como consecuencia que se modifiquen los criterios de diseño y construcción de pavimentos para aeropuertos y que sea necesario evaluar la capacidad de carga de las aeropistas y los métodos constructivos. (Garcia López \& Beltrán Moreno, 1979).

Por lo tanto, es indispensable analizar la infraestructura del trasporte aéreo, en la que las pistas de despegue y aterrizaje ocupan un lugar importante, dado que el estado en el que se encuentre facilita las diferentes maniobras de despegue, aterrizaje, carga y descarga y además, que estas se realicen con cierto grado de seguridad, comodidad, economía y eficiencia. (Murphree, Woodhead, \& Wortman, 1971).

Los aeropuertos en Colombia de tráfico aéreo de pasajeros y de carga más elevado están regulados por la Aeronáutica Civil, que corresponden a 70 de los 202 aeropuertos que actualmente funcionan en el territorio nacional, no obstante, 17 de ellos fueron dados en concesión a empresas privadas buscando que las mismas se encarguen de la administración, modernización y expansión, operación, explotación comercial y mantenimiento del área concesionada, de acuerdo con la información suministrada por la AeroCivil y la ANI, existen en Colombia 6 contratos de concesión mediante los cuales están siendo manejados estos aeropuertos (Durán Preciado, 2013), los cuales se relacionan en la tabla 1:

Tabla 1. Concesiones aeroportuarias en Colombia (2013).

\begin{tabular}{|c|c|c|}
\hline Concesión & Aeropuerto & Ciudad \\
\hline \multirow{6}{*}{$\begin{array}{l}\text { Concesión Aeroportuaria Centro-Norte } \\
\text { AIRPLAN S.A }\end{array}$} & Antonio Roldan Betancourt & Carepa \\
\hline & El Caraño & Quibdó \\
\hline & José María Córdova & Rionegro \\
\hline & Las Brujas & Corozal \\
\hline & Los Garzones & Montería \\
\hline & Olaya Herrera & Medellín \\
\hline AeroCali S.A & Alfonso Bonilla Aragón & Cali \\
\hline Opain S.A & El Dorado & Bogotá D.C \\
\hline $\begin{array}{l}\text { Sociedad Aeroportuaria de la Costa S.a- } \\
\text { SACSA S.A }\end{array}$ & Rafael Núñez & Cartagena de Indias \\
\hline \multirow{2}{*}{$\begin{array}{l}\text { Concesión Aeroportuaria San Andrés y } \\
\text { Providencia S.A -CASYP S.A }\end{array}$} & Gustavo Rojas Pinilla & San Andrés \\
\hline & El Embrujo & Providencia \\
\hline \multirow{6}{*}{ Aeropuertos de Oriente S.A.S } & Palonegro & Bucaramanga \\
\hline & Camilo Daza & Cúcuta \\
\hline & Simón Bolívar & Santa Marta \\
\hline & Yariguires & Barrancabermeja \\
\hline & Almirante Padilla & Riohacha \\
\hline & Alfonso López Pumarejo & Valledupar \\
\hline
\end{tabular}

Fuente: (Duran Preciado, 2013). 
Como se observa en la tabla el concesionario Airplan tiene a su cargo los aeropuertos de la región Centro-Norte de Colombia, incluyendo el aeropuerto Enrique Olaya Herrera, el cual se encuentra ubicado en el Sur Occidente de la ciudad de Medellín y es uno de los aeropuertos con más operaciones aéreas en Colombia, (Aeronautica Civil de Colombia, 2014). En la figura 1 se observa la localización del aeropuerto.

Figura 1. Localización Aeropuerto Enrique Olaya Herrera

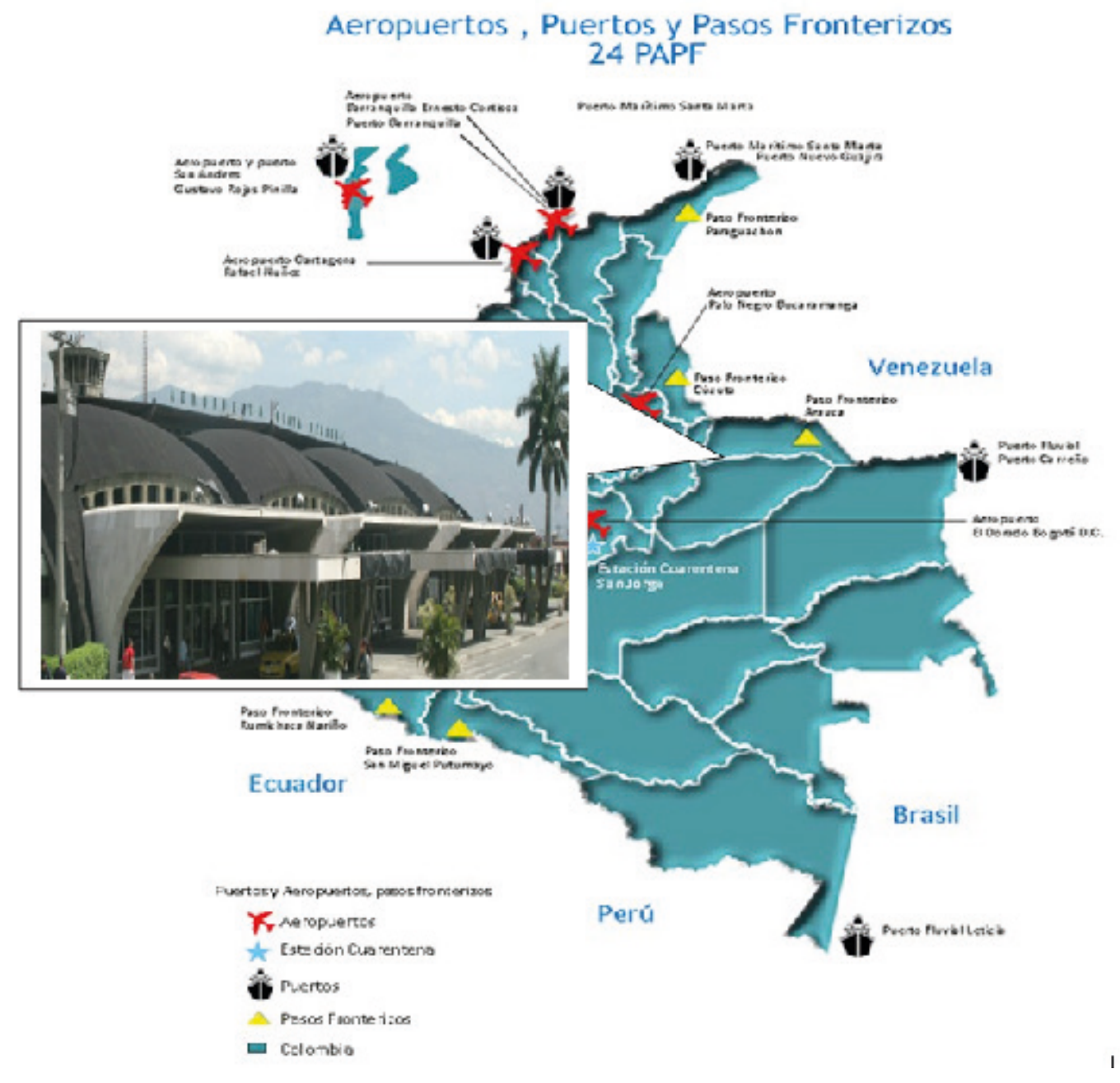

Fuente: Instituto Colombiano Agropecuario, 2014

Dentro de las obligaciones que tiene la concesión se encuentra la de propender por el adecuado desarrollo de las operaciones, lo que está directamente relacionado con la infraestructura, por tal motivo, se contrató una empresa particular para que adelantara las tareas de fresado y repavimentación de la pista principal con miras a obtener un incremento en el índice de estado y más aún mejorar la calificación del Índice de Regularidad Internacional, buscando aumentar el grado de satisfacción de los usuarios y la percepción de seguridad de los mismos al 
utilizar la pista del aeropuerto, generando un incremento en la cantidad de operaciones.

La normativa que rige el proceso de construcción de los aeropuertos en Colombia actualmente es la Federal Aviation Administration (FAA), en su capítulo P 403, contiene las especificaciones de diseño y acabado de la mezcla asfáltica en caliente para carpetas de rodadura, en esta se establece una medida de la regularidad superficial por medio de la regla de tres metros, de manera puntual y no acumulada como sí lo hace el IRI que establece un concepto claro de aceptación o rechazo de los pavimentos construidos antes de la puesta en operación.

El Índice Internacional de Rugosidad (IRI) fue aceptado como estándar de medida de la regularidad superficial de un camino por el Banco Mundial en 1986. Su obtención es posible correlacionarla con cualquier equipo de medición de la rugosidad de un pavimento. (Sánchez \& de Solminihac, 1989).

Durante la ejecución de los trabajos, se evidenció que la norma de la FAA no posee un criterio relacionado con el IRI, razón por la cual el contratista optó por acogerse a esta norma y no regirse por la especificación particular que pretendía lograr una calificación recomendada en los estudios realizados por el Banco Mundial, la cual describía muy claramente que el IRI debería ser inferior a $2 \mathrm{~m} / \mathrm{Km}$, criterio aceptado como una superficie en buen estado y considerando que el pavimento de un aeropuerto tiene el propósito de servir al usuario (el avión, la empresa operadora, el piloto y los ocupantes) proporcionando una serie de condiciones requeridas por este en función de sus necesidades reales y sus deseos. (García López \& Beltrán Moreno, 1979).
Por lo tanto, se pretende adoptar este criterio para avalar o rechazar la calidad del acabado superficial en pistas de aeropuertos, generando una alta calidad en todos los procesos constructivos y asegurando un producto final con excelentes niveles de seguridad y comodidad para los usuarios alargando la vida útil de los pavimentos y reduciendo los costos de mantenimiento de los mismos. Aunque uno de los aspectos más difíciles del análisis del pavimento de las aeropistas es definir cuando deja de ser funcional (Zaniewski, 1991), en la actualidad se admite generalmente que los procedimientos de evaluación de rendimiento funcional del pavimento deben basarse en el análisis de la regularidad, resistencia al deslizamiento, el potencial de daños por objetos extraños a las aeronaves, así como daños en la superficie. En este sentido el Índice Internacional de Regularidad permite especificar rangos o niveles de tolerancia para la aceptación de tramos nuevos de aeropistas y carreteras, sirviendo como un parámetro de control de calidad superficial. (Shain, 1982).

\section{$B$. Aproximación conceptual}

Para comprender claramente el concepto de IRI es necesario abordar las siguientes definiciones:

\section{1) Definición de Rugosidad/Regularidad}

Según el Instituto Nacional de Vías (Invías, 2013) de Colombia, la rugosidad se define como alteraciones del perfil longitudinal de una vía, que provocan vibraciones en los vehículos que lo recorren. Es la desviación de la superficie respecto a una superficie plana, con dimensiones características que afectan la dinámica del vehículo y la calidad al andar. 
Se mide con el indicador denominado Índice de Rugosidad Internacional (IRI), el cual se expresa en $\mathrm{m} / \mathrm{km}, \mathrm{mm} / \mathrm{m}$ o pul $/$ milla.

Es de resaltar que la trascripción exacta de la palabra "Roughness" es rugosidad, término asociado en diferentes países con fricción, pero en el contexto de esta investigación se utilizará el término regularidad superficial como un calificativo más adecuado.

Según Badilla (2009), la regularidad de la superficie de rodadura para la circulación de los vehículos tiene importancia en varios aspectos como son:

\section{a) Seguridad y comodidad:}

Una buena regularidad superficial permite ofrecer condiciones de seguridad y comodidad para los usuarios de las vías.

\section{b) Costos de operación:}

Tiene incidencia en los costos de operación de los vehículos, puesto que, dependiendo de la magnitud de las irregularidades superficiales, la velocidad de circulación puede verse afectada negativamente, lo cual puede reflejarse por un mayor desgaste en llantas y componentes mecánicos de los vehículos y mayor consumo de combustible.

\section{c) Efectos dinámicos:}

Los efectos dinámicos producidos por las irregularidades de las vías, pueden reflejarse no solo en los vehículos, sino también en modificaciones de estado de esfuerzos y deformaciones en la estructura del pavimento, lo que puede incrementar los costos en las actividades de conservación (mantenimiento, rehabilitación o reconstrucción).

\section{d) Acciones de conservación de las vías:}

La regularidad inicial de los pavimentos es un indicador de la calidad de la construcción de las vías. Si este es construido con buena regularidad superficial se espera que su vida útil sea mayor que otro que tenga mayores deformaciones; sin embargo, debe tenerse en cuenta que el progreso de las irregularidades depende de muchos otros factores, como las cargas impuestas por el tráfico, el clima, la variabilidad de los materiales de construcción, el estado de la subrasante, variaciones de topografía, presencia de estructuras en la vía, entre otros aspectos, por lo tanto, es de suma importancia conocer la regularidad superficial del pavimento en cualquier momento desde el inicio de su periodo de servicio o de la vida útil, para definir las acciones de conservación (mantenimiento, rehabilitación o reconstrucción) necesarias en el momento pertinente. (Badilla, 2009).

\section{2) Definición de Índice de Rugosidad Internacional (IRI).}

Se define como la acumulación del movimiento vertical que sufre la suspensión de una rueda (un cuarto de automovil) cuando este recorre la superficie a una velocidad de referencia de 80 $\mathrm{km} / \mathrm{h}$. En este sentido, es un índice de comodidad de rodadura y constituye un parámetro de confort de la vía que percibe el usuario.

Según Caro Rivera \& Peña Castro (2012). Se observó que entre $10 \mathrm{~km} / \mathrm{h}$ y $40 \mathrm{~km} / \mathrm{h}$, el IRI presentaba grandes variaciones en cada tipo de perfil y a partir de $50 \mathrm{~km} / \mathrm{h}$, el comportamiento empezó a ser homogéneo con tendencia a estabilizarse. Por lo anterior, se concluyó que la velocidad de $80 \mathrm{~km} / \mathrm{h}$ con la que fue calibrado el ensayo del Banco Mundial de 1982, es la adecuada para cualquier tipo de pavimento $\mathrm{y}$ puede ser utilizada en la evaluación de aeropistas, ya que este límite de velocidad se supera ampliamente. 


\section{3) Modelo matemático del IRI}

La medición del IRI de un pavimento consiste esencialmente en cuatro pasos (Torrent, 1999):

a) La medición física de un perfil longitudinal simple.

b) Dicho perfil se filtra empleando la media móvil sobre una base de $250 \mathrm{~mm}$ de largo. Este filtrado simula el efecto suavizante de la deformación del neumático.

c)El perfil resultante se vuelve a filtrar mediante la simulación del cuarto de auto. Esta simulación registra la respuesta física de un auto "ideal" que transita sobre el perfil a una velocidad de $80 \mathrm{~km} / \mathrm{h}$. 4. El IRI $(\mathrm{m} / \mathrm{km})$ se calcula como el movimiento acumulado (m) de la suspensión del auto "ideal”, dividido por la longitud del perfil transitado (km). Según Sayers \& Karminhas (1986) el modelo matemático se representa en la figura 2 :

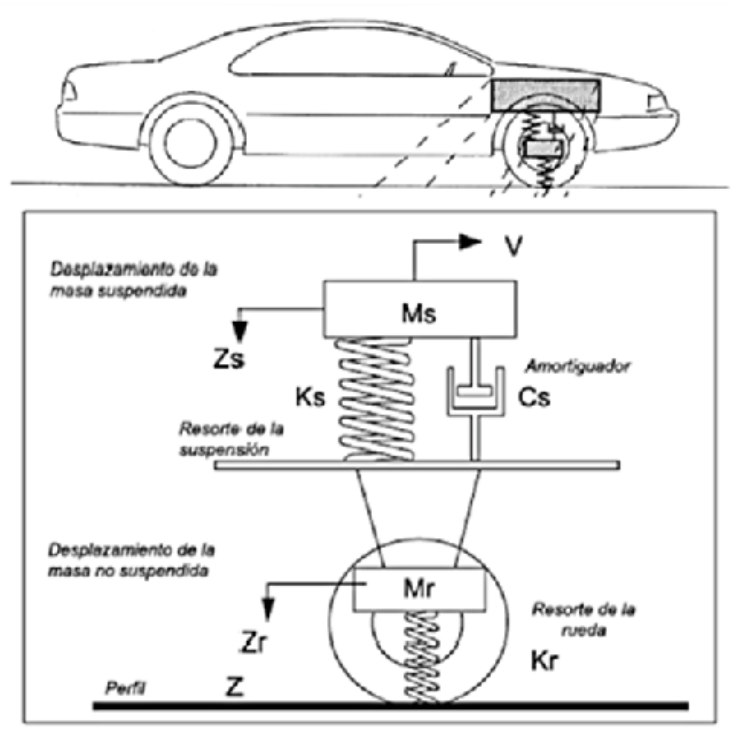

Figura. 2. Representación de masa suspendida y no suspendida en el Modelo del Cuarto de Automóvil.

Fuente: (Badilla, 2009).
Donde:

Ks : Constante del resorte de la suspensión

$\mathrm{Kr}$ : Constante del resorte de la rueda

Ms: Masa suspendida

Mr: Masa no suspendida

Cs: Amortiguador

Por lo anterior, el valor del IRI está definido por ecuación (1):

$$
I R I=\frac{1}{L} \int_{0}^{\frac{X}{V}}\left[Z_{S}-Z_{r}\right] d t
$$

Dónde:

IRI es el Índice de Rugosidad Internacional en $\mathrm{mm} / \mathrm{m}$;

L es la longitud del tramo;

$\mathrm{X}$ es la distancia longitudinal en $\mathrm{m}$;

$\mathrm{V}$ es la velocidad del automóvil modelo $(80 \mathrm{~km} / \mathrm{h})$;

$\mathrm{X} / \mathrm{V}$ es el tiempo que tarda el modelo en recorrer cierta distancia $\mathrm{X}$;

dt es el incremento de tiempo;

ZS es la velocidad vertical de la masa del vehículo;

Zr es la velocidad vertical de la masa del eje.

d) Longitud de onda que incide en el IRI

(Sayers \& Karminhas, 1986) definen que el IRI es influenciado por longitudes de onda que van desde 1.25 hasta 30 metros, a partir del filtro del 
IRI por el modelo del cuarto de automóvil. En la figura 3 , se observa la ganancia o incidencia del perfil en el IRI para diferentes longitudes de onda, en donde la zona en recuadro, corresponde al rango de mayor incidencia, aclarando que existe alguna respuesta seguramente menor para las longitudes de onda que están por fuera del rango.

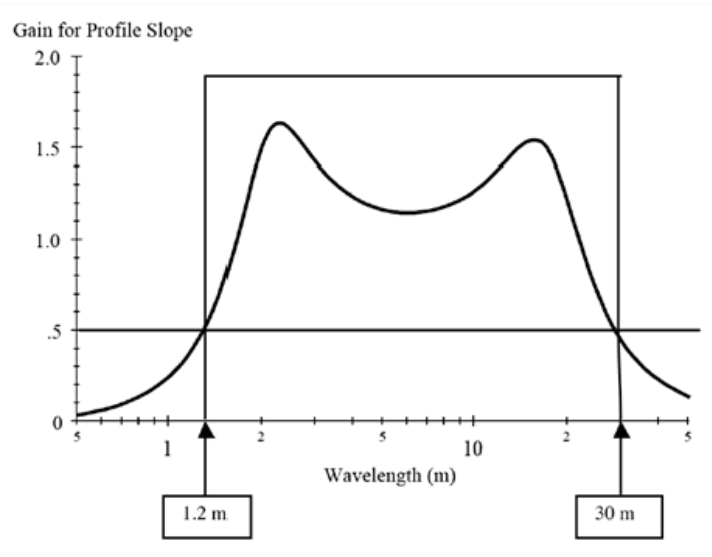

Figura. 3. Representación gráfica de la influencia a del IRI para diferentes longitudes de onda.

Fuente: (Sayers \& Karminhas, 1986).

En la figura 3 se observan dos zonas que están por fuera del rango (1.20m-30m), la zona inferior o pequeñas longitudes de onda corresponde a irregularidades propias de microtexturizados, macrotexturizados y megatexturizado, cuyo aporte en la evaluación del IRI es mínimo, pero no despreciable del todo, mientras que la zona con longitudes de onda mayores a $30 \mathrm{~m}$ corresponde a ondas largas que bien pueden ser propias de la geometría de la vía, como curvas verticales y demás aspectos propios de la topografía de la vía, que tampoco reflejan valores de IRI considerables según el filtro del modelo. Las irregularidades que mayor incidencia tienen en el IRI son aquellas de longitud de onda corta y media y algunas de onda larga, comprendidas entre $1.25 \mathrm{~m}$ y $30 \mathrm{~m}$, y estas pueden corresponder a juntas, bacheos irregulares, depresiones, fallos, hundimientos, ondulaciones, curvas verticales y presencia de estructuras hidráulicas, entre otras. (Caro Rivera \& Peña Castro, 2012).

Para calcular el IRI es necesario conocer los siguientes elementos:

- Perfil longitudinal de la carretera: El perfil de la carretera se define como las cotas en intervalos de longitud constante, mayor de $0.01 \mathrm{~m}$ y no más de $0.3 \mathrm{~m}$.

- Desplazamiento vertical, $u$ : Desplazamiento vertical de las masas no suspendidas, con relación a su posición inicial.

- Desplazamientovertical, $v$ : Desplazamiento vertical de las masas suspendidas, con relación a su posición inicial.

- Longitud de valoración del IRI, L: Longitud del tramo en el que se calculará el IRI y se reportará un resultado. Para los cálculos que siguen en esta norma, la longitud se considerará igual a $100 \mathrm{~m}$. (Caro Rivera \& Peña Castro, 2012).

\section{Resultados y análisis}

La práctica habitual en la pavimentación con mezclas asfálticas en caliente, busca establecer un paquete estructural multicapa que soporte los esfuerzos actuantes por parte de los vehículos que circulan sobre esta estructura. Bajo estas premisas, se construyeron gran parte de las vías de nuestro país y las pistas para aeropuertos no fueron la excepción. Pero existen otros aspectos a considerar en el diseño y construcción de un 
pavimento indiferente del tipo de vehículo que circulará por él y el número de repeticiones esperadas o tránsito, tal es el caso de la regularidad superficial (IRI), la macrotextura, el ahuellamiento, características de deterioro superficial asociadas a la seguridad y el confort que experimenta el usuario al rodar por este nuevo pavimento. Estas características no son solamente un indicador de comodidad, sino que también pueden ser utilizados para evaluar y cuantificar el costo de uso de las estructuras y así obtener pavimentos con mejores estándares de calidad.

Aunque el Índice de Regularidad Superficial (IRI), no es el único criterio de auscultación de un pavimento ya sea flexible o rígido, sí es uno de los parámetros de mayor aceptación a nivel mundial como indicativo de deterioro superficial y estructural de un firme. La auscultación de vías en nuestro país a través del sistema de concesiones ha generado un impacto positivo, al adoptar estos criterios internacionales para la aceptación o rechazo de un pavimento recientemente construido, lo que implica adoptar un esquema de construcción muy cuidadoso que genere un producto con la calidad esperada por los usuarios y soportada por medio de calificaciones como la del IRI. Las concesiones aeroportuarias, no deben estar al margen con la utilización de la auscultación de sus pistas, así el organismo internacional que ampara la construcción de las aeropistas (FAA), no haga énfasis en este tipo de análisis de calidad para la recepción de los pavimentos nuevos o repavimentaciones.

Se podría pensar que una carretera no es comparable con una aeropista, ya que su velocidad y alineamiento son muy diferentes, así sean estructuras asfálticas similares en cuanto a su composición y textura, variando solamente en los espesores utilizados. Pero tal como se demostró en la experiencia del Banco Mundial en 1986 y lo establecen otros estudios como el de Caro Rivera \& Peña Castro (2012) en el que se observaron comportamientos homogéneos en la medición del IRI a partir de los $80 \mathrm{Km} / \mathrm{h}$, lo cual hace que el parámetro sea utilizable en pistas de aeropuertos como indicativo de calidad y sinónimo de seguridad de las mismas.

El IRI como criterio de aceptación de la infraestructura aeroportuaria (pistas, calles de rodaje y plataformas), debe ser establecido contractualmenteparapromoverelmejoramiento de los procesos constructivos antes de la entrega del producto final, incrementando los niveles de seguridad y calidad tanto de la estructura del pavimento como del acabado superficial o macrotextura dado que existe una relación exponencial entre la ocurrencia de accidentes y valores bajos de rugosidad, para que con esta información el concesionario programe las acciones preventivas de conservación ahorrando millones de dólares en reparaciones que se pudieron prevenir con intervenciones oportunas menos costosas.

Tal como lo afirman Garcia López \& Beltrán Moreno (1979), se requiere entonces contar con técnicas convenientes de evaluación que hagan posible decidir sobre las últimas actividades y desarrollar, comprobar o ajustar métodos de diseño y evaluación suficientemente amplios y prácticos para cubrir el balance económico avión-pavimento, dualidad que se genera al utilizar la pista en buenas o regulares condiciones lo que implica daños mecánicos, cierres temporales y sobrecostos en las operaciones que pueden ser minimizados utilizando un indicador de la calidad del pavimento como lo es el Índice de Regularidad Internacional y posibilitando una adecuada planificación del mantenimiento y conservación de la infraestructura aeroportuaria. 
Desde el punto de vista de la gestión de pavimentos, la obtención de un buen Índice de Regularidad Internacional garantiza buenos procesos constructivos, durabilidad de las estructuras de pavimento, optimización de los recursos de mantenimiento, seguridad al utilizar las pistas en cualquier condición climática y comodidad tanto de los pasajeros como de la tripulación de las aeronaves; además permite tener una visión más global del deterioro del pavimento, pues no se limita al estudio de un tramo en particular sino que hace una evaluación total del estado de las estructuras ampliando de esta forma el espacio de inferencia del método.

\section{Conclusiones}

Se ha demostrado la aplicabilidad del índice de regularidad internacional para evaluar el estado funcional de cualquier pavimento flexible o rígido, para automóviles o aviones en este caso.

La incorporación de este parámetro (IRI) dentro del contrato inicial como criterio de aceptación de las aeropistas en concesión es fundamental para mejorar las condiciones de acabado y comodidad, así como elevar el nivel de percepción de seguridad de los usuarios.

La seguridad es el pilar de las operaciones aéreas, la percepción que los usuarios tengan de esta incentivara el uso de este medio de transporte, traduciéndose este hecho en un beneficio económico para todos.

Estudios realizados a nivel internacional demuestran que la velocidad no es un factor excluyente para la implementación de este análisis, dado que un avión circula a velocidades muy superiores a las de cualquier automóvil en condiciones normales.
El IRI como criterio de aceptación de la infraestructura aeroportuaria (pistas, calles de rodaje y plataformas), debe ser establecido contractualmenteparapromoverelmejoramiento de los procesos constructivos antes de la entrega del producto final, incrementando los niveles de seguridad y calidad tanto de la estructura del pavimento como del acabado superficial o macrotextura.

Los costos de operación de un vehículo son directamente proporcionales a las irregularidades superficiales de los pavimentos y se comportan de acuerdo a la magnitud del vehículo; así mismo ocasionan deformaciones en la estructura del pavimento, incrementando los costos de mantenimiento y rehabilitación.

Es de resaltar que cuando un avión se enfrenta a un pavimento en mal estado no puede simplemente como los automóviles reducir la velocidad para maniobrar, ya que este debe alcanzar una cierta velocidad tanto para despegar como para aterrizar. Una pista en mal estado aumenta exponencialmente el riesgo de accidentalidad con todas sus repercusiones económicas y sociales.

Otro aspecto importante relacionado con la frecuencia de mantenimiento de una aeropista, es el de cierre de la misma, lo cual impone serias dificultades tanto económicas como de operación que se pueden distanciar en el tiempo obteniendo un buen Índice de Regularidad Internacional.

El éxito de construir un pavimento de calidad, no está dado solamente por un buen diseño y adecuada preparación de los materiales. También es muy importante una adecuada colocación y compactación de todas las capas de la estructura, lo cual finalmente nos dará una calificación buena en términos de IRI redundando en una estructura cómoda y con bajos costos de mantenimiento. 
Es importante que todas las personas que de uno u otro modo interactúan durante la construcción de un pavimento, comprendan las variables más relevantes que inciden en la vida útil, el acabado y los costos de mantenimiento, para que de una manera consiente se preocupen por las buenas prácticas constructivas y se incremente la durabilidad de una vía o aeropista.

Un pavimento en buenas condiciones, o sea con un índice de regularidad dentro de los $2 \mathrm{~m} / \mathrm{Km}$, garantiza operaciones de transición aire-tierra-aire más cómodas y seguras tanto para los usuarios de las aeronaves como para la tripulación misma, generando un estado de satisfacción en la comunidad que se traduce en mayores índices de uso de este medio de transporte.

\section{Referencias bibliográficas}

Aeronaútica Civil de Colombia. (2014). Origen-Destino. Tomado de: $\underline{\text { http:// }}$ www.aerocivil.gov.co/atencion/estadisticas-de-las-actividades-aeronauticas/ Estadsticas\%20operacionales/Boletin\%20 Estadistico\%20Febrero\%202006.pdf

Air Transport Action Group. (2012).
$\begin{aligned} & \text { Aviation benefits beyond borders. } \\ & \text { Tomado de: }\end{aligned}$
fw-nottp://passthrough.net/download/474243/http://
aviationbenefits.org/media/26786/ATAG
AviationBenefits2014 FULL_LowRes.pdf

Badilla, G. (2009). Determinación de la regularidad superficial del pavimento, mediante el cálculo del Índice de Regularidad Internacional (IRI). Infraestructura Vial, $\mathrm{N}^{\circ} 21.30-37$.

Caro Rivera, F \& Peña Castro , G. (2012). Analisis y Criterios para el cálculo del indice de rugosidad internacional(IRI) en vías urbanas Colombianas que Orienten la elaboracion de una especifiacion tecnica. Intekhnia, 7(1).
Duque Escobar, G. (2007). Fundamentos de economía y transporte. Tomado de: http:// www.bdigital.unal.edu.co/1879/5/03-intec-transp.pdf

Durán, D. (2013). Estudios de Mercado Aeropuertos de Colombia. Tomado de: http://www. sic.gov.co/recursos user/documentos/ promocion competencia/Estudios Economicos/Estudios Economicos/ Estudios_Mercado_Aeropuertos.pdf

Federal Aviation Administration. (Julio de 21 de 2014). Airport Construction Standards Tomado de: http://www.faa.gov/airports/ engineering/construction_standards/

García, M. (1979, octubre). Evaluación de pavimentos para aeropuertos. Ponencia presentada en el Tercer Simposio Colombiano sobre Ingeniería, Cartagena, Colombia.

Instituto Colombiano Agropecuario. (2014). Tomado de http://www.ica.gov.co/

Monischmit, C. (Abril de 1977). Air Transportation Division Specialty Conference. Washington, D.C., United States.

Murphree, E., Woodhead, R. \& Wortman,R. (1971). Airfild Pavement Systems. Transportation Engineering Journal.

Olivera, M., Cabrera, P., Bermúdez, W \& Hernández, A. (2011). El Impacto del transporte aéreo en la economía Colombiana y las políticas públicas. Bogotá: Fedesarrollo.

Sánchez, I., \& de Solminihac, H. (1989) El IRI: un indicador de la regularidad superficial. Revista de Ingeniería de Construcción, (6), $1-17$.

Sayers, M., \& Karminhas, S. (1986). Interpretation of Road Roughness Profile Data Federal Highway Administration. Report Word Bank Technical Paper No.45. 96-101. Tomado de: http://citeseerx.ist.psu.edu/viewdoc/do 
wnload?doi=10.1.1.40.5876\&rep=rep1\&ty pe $=$ pdf

Shain, M. (1982). Aeródromo: Medidas de fatiga del pavimento y su utilización en la gestión de pavimentos. Transportation Research Record. Tomado de: http://www. trb.org/Main/Blurbs/154702.aspx

Torrent, R. (1999). Avances en la Rugosidad (IRI) de Pavimentos de Hormigón Construidos con Pavimentadoras de Molde Deslizante. Buenos Aires,: Instituto del Cemento Portland Argentino. Tomado de: https:// xa.yimg.com $/ \mathrm{kq} /$ groups $/ 13240622 / \ldots /$ name/Avances + en + la + rugosidad.doc

Witczak, M. (1978). Framework for evaluation and performance of airport pavements. Transportation Research Board. Tomado de: $\quad$ http://www.trb.org/Main/Search2. aspx?q 